NOTA TECNICA

\title{
EFECTOS DE APTITUD COMBINATORIA GENERAL E IDENTIFICACIÓN DE HÍBRIDOS TRIPLES DE MAÍZ (Zea mays L) GRANO BLANCO. PROGRAMA REGIONAL DE MAÍZ (PRM), 1991.*
}

\author{
Mario Fuentes**, Jose Luis Quemé*** y Carlos Pérez***
}

\begin{abstract}
RESUMEN
Se evaluó la formación de 91 cruzas triples con la combinación de 24 líneas de grano blanco y cuatro cruzas simples élite utilizadas como hembra en la formación de los híbridos HB-83M, H-5, H-27 y DC-43 de Guatemala, El Salvador, Honduras y Costa Rica, respectivamente, con el objetivo de determinar la aptitud combinatoria general ACG del germoplasma desarrollado dentro del Proyecto Regional de Maíz, las cuales se evaluaron en 10 localidades de Centro América.

Los resultados demuestran una superioridad notable en el comportamiento de los nuevos híbridos formados, comparados con los testigos HB-83, H-5, H-27, HC-43 Y los probadores utilizados. El mayor rendimiento se obtuvo en las localidades de Costa Rica, con el híbrido (1188x1178) con 8.60 t/ha y superó al testigo HC-43 en $35 \%$.

Las estimaciones de ACG identificaron a 9 líneas superiores, dentro de las cuales sobresalen la 1178, 1107 y 1130 con valores de 6,2, 6,1 y 6,0 t/ha, derivadas del Pool 24 y de la Población 25 de CIMMYT.
\end{abstract}

\begin{abstract}
Effect of Overall Combining Aptitude aod Identification of Triple Hybrids of White Grain Corn (Zea mays L.). The breeding of triple crosses with the combination of 24 white grain lines and four elite simple crosses, used as females in the breeding of the hybrids HB-83M, H-5, H-27 and DC-43 in Guatemala, El Salvador, Honduras and Costa Rica, respectively, were evaluated in ten localities in Central America to determine the overall combining ability OCA of the germplasm developed by the Regional Corn Project.

The results show an out standing superiority in the perfomance of the new hybrids bred, compared to the controls HB-83, H-5, H-27, HC-43, and the testers used. The highest yields were obtained at the Costa Rican localities with the hybrid $1188 \times 1178$ with $8.6 \mathrm{t} / \mathrm{ha}$, and overyielded the control HC-43 by $35 \%$.

The OCA estimated identified 9 superior lines, among which the 1178,1107 , and 1150 stand out with values of $6,2,6,1$ and 6,0 t/ha, derived from the Pool 24 and the Population 25 from CYMMYT.
\end{abstract}

\section{INTRODUCCION}

En Centro América y El Caribe, se siembran más de dos millones de hectáreas de maíz (CIMMYT, 1990), y se constituye uno de los cereales de mayor importancia por su utilización en la alimentación humana, animal y en algunos casos para la industria. En 1986, se establecieron proyectos colaborativos de investigación en maíz entre el CIMMYT y los Programas Nacionales de Maíz, tendientes a establecer estrategias para encontrar soluciones a la problemática que limita la producción y productividad de dicho cultivo. Guatemala es el líder en el Proyecto Regional de Híbridos de maíz y una de las fases importantes en éste proyecto lo constituye el

\footnotetext{
* Trabajo presentado en la XXXVIII Reunión Anual del PCCMCA, Managua, Nicaragua, 23-27 de marzo de 1992.

** Coordinador Programa de Maiz. lerA Guatemala.

*** Técnicos Programa de Ma i z. ICTA. Guatemala.
} 
mejoramiento de los híbridos en actual producción comercial en los diferentes países.

Los objetivos del presente trabajo fueron: 1) evaluar híbridos trilineales grano blanco con endogamia parcial, utilizando como probadores distintas cruzas simples élite desarrolladas por los Programas Nacionales de Guatemala, El Salvador, Honduras y Costa Rica; 2) determinar la aptitud combinatoria general de líneas élite desarrolladas por el Proyecto Regional de híbridos de maíz; 3) identificar el híbrido trilineal superior con mejor respuesta a las áreas de potencial de producción en Centro América y específica para cada país.

La diversidad gen ética y aptitud combinatoria son componentes importantes para obtener altos valores de heterósis en la formación de híbridos de maíz. La aptitud combinatoria general proporciona información sobre que lineas puras deben producir los mejores híbridos cuando se cruzan con muchas otras líneas, asi mismo se ha definido como el valor medio F1' s de sus cruzas con otras líneas (Jugenheimer, 1981). Vasal et al., citado por Quemé et al. (1990), menciona que según estudio del Programa de Economía del CIMMYT en 1986, los híbridos ocuparon el 36\% del área total dedicada al cultivo de maíz en el tercer mundo, y el incremento de agricultores capaces de adoptar híbridos, ha provocado el interés de varios programas nacionales por la creación de híbridos. El Programa de Maíz del CIMMYT inició su propio programa de híbridos con objetivos generales de generar nuevos tipos de germoplasma para que se utilicen en la generación de híbridos y además dar información relacionada con la utilidad de los materiales tropicales y subtropicales.

Según Brauer (1980), el método clásico para la formación de híbridos consiste en desarrollar lineas puras por medio de endogamia y selección continua durante varias generaciones, hasta lograr líneas con suficiente homocigosis que presentan características deseables. Douglas (1982), menciona que el éxito del mejoramiento genético está determinado por el producto final, en nuestro caso será el híbrido. Allard (1980) y Brauer (1980), indican que con el mejoramiento genético se logra valorizar a las líneas progenitoras de los híbridos mediante las pruebas de aptitud combinatoria general (ACG) y específica (ACE) logrando con ello determinar la capacidad de las líneas para producir híbridos superiores cuando se cruzan con otras líneas. (Quemé et al. 1990).
Los Programas Nacionales de Maíz en la región han desarrollado en sus propios países proyectos de mejoramiento, con el fin de generar variedades e híbridos con potencial de rendimiento, mejores caracteristicas agronómicas, amplia adaptación y otros caracteres específicos para cada país; sin embargo, los logros obtenidos no han sido similares en cada uno de los paises (CIMMYT, 1989). Las cruzas simples utilizadas como hembras en los híbridos se usan como probadores para definir la aptitud combinatoria de las líneas élite en generaciones tempranas (S3) desarrolladas por el Proyecto Regional de Maíz y los híbridos triples formados a partir de dichas cruzas se evaluan en diferentes localidades e identifican los superiores en función del rendimiento y características agronómicas.

\section{MATERIALES Y METODOS}

\section{Localización}

La evaluación se realizó en 10 localidades de Centro América; San Jerónimo, La Máquina, Cuyuta y Las Vegas en Guatemala, San Andrés y Santa Cruz Porrillo en El Salvador, Omonita en Honduras, San Cristobal en Nicaragua y dos localidades en Alajuela, Costa Rica.

\section{Material genético}

El germoplasma utilizado como probadores fueron las cruzas simples hembras de los híbridos: HB-83M, H27, H-5 y DC-43, originarias de Guatemala, Honduras, El Salvador y Costa Rica, que se identifican en la evaluación como $1185,1186,1187$ y 1188, respectivamente, las cuáles se mestizaron con 24 líneas élite derivadas de las poblaciones 22, 25, 32,49 y Pool 24 de CIMMYT.

\section{Diseño experimental}

Se utilizó látice simple 10x10 con 100 tratamientos y 2 repeticiones por localidad.

\section{Fecha de siembra y cosecha}

La fecha se siembra fué en junio y la cosecha se realizó en octubre. Las distancias de siembra fué de 0.75 $\mathrm{m}$ entre surcos y $0.50 \mathrm{~m}$ entre postura de 2 plantas, equivalente a 53,000 plantas por hectárea, el manejo agronómico se realizó de acuerdo a la tecnología de cada 
país. La parcela experimental consistió en dos surcos de $5,50 \mathrm{~m}$ de largo con una parcela útil de $8.25 \mathrm{~m}^{2}$.

Las variables estudiadas fueron: Rendimiento, días a floración, altura de planta y mazorca, porciento de mazorca descubierta y podrida, porcentaje de acame de raíz y tallo.

\section{Análisis estadístico}

Se realizó análisis de varianza por localidad bajo el modelo de látice para la variable rendimiento, la comparacion de medias se realizo por medio de la prueba de Tukey y la identificación de las líneas con mejor aptitud combinatoria general se realizó a través de los cuatro probadores utilizados.

\section{RESULTADOS Y DISCUSION}

El Cuadro 1, presenta los estadísticos estimados en el análisis de varianza para rendimiento por localidad y combinado, la alta significancia para tratamientos e interacción genotipo por localidad, indica que existe una respuesta diferencial para el rendimiento en los genotipos evaluados y presentan una respuesta relativamente diferente en algunas localidades.

Cuadro 1. Estadísticos estimados en el análisis de varianza para rendimiento de los mestizos de maíz blanco del Programa Regional de Maíz. Centro América PRM, 1991.

\begin{tabular}{|c|c|c|c|c|c|}
\hline País & Localidad & $\begin{array}{r}\text { Media } \\
t / h a\end{array}$ & $\begin{array}{l}\text { DMS } \\
\text { t/ha }\end{array}$ & $\stackrel{\%}{\text { C.V. }}$ & $\begin{array}{c}\text { F trat } \\
0,01\end{array}$ \\
\hline Guatemala & San Jerónimo & 8,170 & 0,980 & 7 & $\star \star$ \\
\hline Guatemala & La Máquina & 1,900 & 1,280 & 34 & $\star \star$ \\
\hline Guatemala & Cuyuta & 6,560 & 1,550 & 12 & $\star \star$ \\
\hline Guatemala & Las Vegas & 5,300 & 1,320 & 13 & $\star \star$ \\
\hline El Salvador & - San Andrés & 4,880 & 1,270 & 13 & $\star \star$ \\
\hline El Salvador & - Santa Cruz & 4,840 & 1,670 & 18 & $\star \star$ \\
\hline Honduras & Omonita & 6,120 & 1,200 & 10 & * \\
\hline Nicaragua & San Cristóbal & 4,310 & 1,080 & 13 & $\star \star$ \\
\hline Costa Rica & Alajuela I & 5,690 & 1,960 & 18 & $\star \star$ \\
\hline Costa Rica & Alajuela II & 8,490 & 0,240 & 2 & $\star \star$ \\
\hline Combinado & & 5,630 & 0,520 & 4 & $\star \star$ \\
\hline
\end{tabular}

* Significativo $5 \%$

** Significativo $1 \%$

Los coeficientes de variación oscilaron entre $2 \%$ para la localidad de Alajuela 11, Costa Rica y 34\% en La Máquina, Guatemala. Las localidades de San Jerónimo, Guatemala y Alajuela 11, Costa Rica, fueron los ambientes con mayor potencial de rendimiento con $8.17 \mathrm{y}$ 8.49 t/ha y la localidad de La Máquina, Guatemala, la de menor rendimiento con $1.90 \mathrm{t} / \mathrm{ha}$. en esta localidad se presentaron problemas de sequía en la época de floración que afectó la expresión del potencial del rendimiento.

El Cuadro 2, presenta los híbridos superiores de maíz obtenidos de la evaluación en los diferentes países participantes. A traves de 4 localidades en Guatemala con el probador cruza simple hembra del HB-83, sobresalen los híbridos trilineales: $(1185 \times 1178)$ y $(1185 \times 1130)$ con 6.40 y $6.35 \mathrm{t} / \mathrm{ha}$ respectivamente, comparado con el híbrido testigo HB-83 que obtuvo 5.24 t/ha y lo superan hasta en $22 \%$ de rendimiento. Las características agronómicas se consideran aceptables y reviste mucha importancia los bajos porcientos de mazorca descubierta (2-8\%) y pudrición de mazorca (4-10\%) que expresan los materiales en evaluación, lo cuál indica que existen genotipos tolerantes a este factor adverso de orígen biótico, si se considera que pudrición de mazorca es la enfermedad de mayor importancia económica en Honduras, Costa Rica y Guatemala, con pérdidas anuales hasta el 20\% (Cordova, 1990).

En los híbridos superiores para Honduras que fueron cruzados con el probador hembra H-29; el mayor rendimiento lo expresó el híbrido (1186xl107) con 8.18 t/ha comparado con el testigo comercial H-29 que rindió $6.10 \mathrm{t} /$ ha $\mathrm{y}$ lo supera en $34 \%$, la expresión de características de calidad de mazorca (cobertura, pudrición) son notables.

La evaluación en El Salvador a través de las localidades de San Andrés y Santa Cruz Porrillo muestran los híbridos superiores de maíz que fueron cruzados con el probador hembra del $\mathrm{H}-5$. El potencial de rendimiento del híbrido (1187x1123) y (1187x1152) es de 5.38 y 5.31 t/ha, respectivamente y superan hasta en $54 \%$ al híbrido comercial H-5 que obtuvo 3.49 t/ha. En general las características agronómicas de los híbridos seleccionados para este país se consideran aceptables.

La evaluación en las localidades de Costa Rica es un ambiente propicio para el desarrollo del complejo de pudrición de mazorca y tallo, causada por los hongos Diplodia sp. y Fusarium sp. Se utilizó como probador hembra la cruza simple DC-43, los híbridos evaluados expresan rendimientos entre 8.60 y $7.81 \mathrm{t} / \mathrm{ha}$. entre los que sobresale el $(1188 \times 1178)$ con $8.60 \mathrm{t} /$ ha y supera en $35 \%$ de rendimiento al híbrido comercial HC-43, testigo 
Cuadro 2. Rendimiento y caraterísticas agronómicas de híbridos triples superiores de maíz, grano blanco evaluados en las diferentes localidades de Centro America. Guatemala, PRM, 1991.

\begin{tabular}{|c|c|c|c|c|c|c|c|c|c|}
\hline HIBRIDO & $\begin{array}{l}\text { Rend. } \\
\text { t/ha }\end{array}$ & $\begin{array}{l}\% s / \\
\text { test. }\end{array}$ & $\begin{array}{l}\text { Dias } \\
\text { flor }\end{array}$ & $\begin{array}{l}\text { Altura } \\
\text { Planta }\end{array}$ & $\begin{array}{l}\text { Altura } \\
\text { Mazorca }\end{array}$ & $\begin{array}{c}\% \\
\text { Desc. }\end{array}$ & $\begin{array}{l}\text { Mazorca } \\
\text { Podrida }\end{array}$ & $\begin{array}{l}\% \\
\text { Raíz }\end{array}$ & $\begin{array}{l}\text { Acame } \\
\text { Tallo }\end{array}$ \\
\hline \multicolumn{10}{|l|}{ GUATEMALA } \\
\hline $1185 \times 1178$ & 6,40 & 122 & 56 & 248 & 141 & 2 & 6 & 6 & 3 \\
\hline $1185 \times 1130$ & 6,35 & 121 & 57 & 243 & 126 & 3 & 4 & 2 & 0 \\
\hline $1185 \times 1111$ & 6,17 & 118 & 58 & 240 & 123 & 8 & 10 & 7 & 0 \\
\hline HB-83* & 5,24 & 100 & 60 & 238 & 127 & 5 & 8 & 7 & 0 \\
\hline HB- $83 * * k$ & 4,86 & & 58 & 238 & 127 & 5 & 7 & 7 & 0 \\
\hline \multicolumn{10}{|l|}{ HONDURAS } \\
\hline $1186 \times 1107$ & 8,18 & 134 & 54 & 228 & 127 & 1 & 0 & 0 & 0 \\
\hline $1186 \times 1111$ & 6,85 & 112 & 55 & 214 & 125 & 1 & 0 & 0 & 1 \\
\hline $1186 \times 1124$ & 6,84 & 112 & 54 & 197 & 110 & 1 & 0 & 0 & 2 \\
\hline$H-29 *$ & 6,10 & 100 & 53 & 222 & 134 & 1 & 1 & 0 & 0 \\
\hline$H-29 * \star$ & 5,58 & & 60 & 243 & 137 & 3 & 15 & 17 & 1 \\
\hline \multicolumn{10}{|l|}{ SALVADOR } \\
\hline $1187 \times 1123$ & 5,38 & 154 & 56 & 218 & 127 & 1 & 2 & 3 & 1 \\
\hline $1187 \times 1152$ & 5,31 & 152 & 60 & 253 & 165 & 4 & 4 & 6 & 5 \\
\hline $1187 \times 1155$ & 5,17 & 148 & 59 & 249 & 138 & 5 & 1 & 1 & 1 \\
\hline $\mathrm{H}-5^{*}$ & 3,49 & 100 & 61 & 241 & 129 & 2 & 1 & 2 & 2 \\
\hline$H-5 * \star$ & 4,29 & & 60 & 258 & 153 & 1 & 6 & 17 & 1 \\
\hline \multicolumn{10}{|c|}{ COSTA RICA } \\
\hline $1188 \times 1178$ & 8,60 & 135 & 58 & 212 & 121 & 2 & 5 & 24 & 0 \\
\hline $1188 \times 1155$ & 8,30 & 130 & 58 & 238 & 125 & 4 & 4 & 42 & 0 \\
\hline $1188 \times 1146$ & 7,90 & 124 & 57 & 212 & 110 & 1 & 6 & 32 & 0 \\
\hline $\mathrm{HC}-43^{\star}$ & 6,38 & 100 & 59 & 225 & 112 & 5 & 8 & 23 & 2 \\
\hline$H C-43 * *$ & 4,62 & & 59 & 225 & 124 & 10 & 8 & 3 & 1 \\
\hline \multicolumn{10}{|l|}{ NICARAGUA } \\
\hline $1186 \times 1146$ & 5,76 & 138 & 54 & 215 & 115 & & 6 & & \\
\hline $1186 \times 1155$ & 5,74 & 137 & 55 & 219 & 116 & & 4 & & \\
\hline $1185 \times 1155$ & 5,68 & 133 & 54 & 225 & 118 & & 8 & & \\
\hline $\mathrm{H}-5$ * & 4,18 & 100 & 57 & 223 & 119 & & 4 & & \\
\hline
\end{tabular}

* Media de evaluacion local.

** Media de evaluación regional.

que produjo $6.38 \mathrm{t} / \mathrm{ha}$, la tolerancia al complejo de pudrición de mazorca manifestada por los híbridos en evaluación bajo estas condiciones de factor adverso biótico son notables, los cuáles oscilan entre 4-8\%.

La evaluación en Nicaragua, presenta un ambiente propicio para el desarrollo del achaparramiento del maíz, factor biótico de importancia económica cuyo vector es al insecto Dalbulus maydis.

En el Cuadro 3 están los híbridos superiores en Nicaragua, los cuales expresaron rendimiento de hasta 5.76 t/ha para el híbrido (1186x1146), comparado con el testigo comercial H-5 que obtuvo $4.18 \mathrm{tm} / \mathrm{ha}$ el cual ha sido un híbrido utilizado como comparador regional y lo supera hasta en un 38\%. En general los híbridos superiores presentan por ciento de achaparramiento de planta entre $20-32 \%$ y por ciento de achaparramiento de mazorca entre $3-28 \%$. Estos valores de achaparramiento son relevantes al compararlos con variedades susceptibles que manifiestan hasta 53\% de mazorcas afectadas (CIMMYT, 1990).

En el Cuadro 4, se observan los rendimientos promedios de la combinación de las mejores líneas con diferentes probadores y superan en su mayoría a las cruzas simples con rendimientos entre 5.76 y 6.20 t/ha así como a los híbridos testigos de la evaluación a excepción de la cruza hembra del HB- 83 que rindió 6.61 t/ha. Las características agronómicas se mantienen similares y las cruzas donde se incluyen las líneas seleccionadas presentan bajos valores de mazorca descubiertas y podridas entre los que sobresalen 1178 y 1107. 
La estimación de aptitud combinatoria general de las líneas superiores a través de los diferentes probadores se presentan en el Cuadro 5 y obtuvieron valores positivos entre 0.17-0.61 t/ha. Se seleccionaron 9 líneas superiores entre las que sobresalen las líneas 1178 derivada del Pool 24, 1107 y 1130 de la población 25, 1152, 1130, 1146 y 1147 con tolerancia al achaparramiento y 1111 de la Población 49, respectivamente. La línea que mejor combinación híbrida mostró fué la 1178 con ACG de 0.61 $\mathrm{t} / \mathrm{ha}$ y media de rendimiento de $6.20 \mathrm{tm} / \mathrm{ha}$.
Los Programas Nacionales pueden hacer uso efectivo de las líneas élite para desarrollar nuevas combinaciones híbridas entre ellas o para producir en forma inmediata los híbridos triples.

\section{CONCLUSIONES}

Se identificaron hibridos triples para Guatemala, El Salvador, Honduras y Costa Rica, con rendimiento que oscilan entre 5.38 y $8.60 \mathrm{t} / \mathrm{ha}$, los que superan a los

Cuadro 3. Rendimiento y características agronómicas de híbridos triples grano blanco, evaluados en Nicaragua. PRM, 1991.

\begin{tabular}{|c|c|c|c|c|c|c|c|c|}
\hline Híbrido & $\begin{array}{l}\text { Rend. } \\
t / \text { ha }\end{array}$ & $\begin{array}{l}\% \text { s/ } \\
\text { test. }\end{array}$ & $\begin{array}{l}\text { Días } \\
\text { flor }\end{array}$ & $\begin{array}{l}\text { Altura } \\
\text { planta }\end{array}$ & $\begin{array}{l}\text { Altura } \\
\text { Mazorca }\end{array}$ & $\begin{array}{l}\text { \%Mazorca } \\
\text { Podridas }\end{array}$ & $\begin{array}{l}\text { \%Achap. } \\
\text { Planta }\end{array}$ & $\begin{array}{l}\text { \%Achap. } \\
\text { Mazorca }\end{array}$ \\
\hline $1186 \times 1146$ & 5,76 & 138 & 54 & 215 & 115 & 6 & 24 & 9 \\
\hline $1186 \times 1155$ & 5,74 & 137 & 55 & 219 & 116 & 4 & 23 & 10 \\
\hline $1185 \times 1155$ & 5,68 & 133 & 54 & 225 & 118 & 8 & 21 & 17 \\
\hline $1186 \times 1147$ & 5,63 & 135 & 54 & 218 & 117 & 17 & 21 & 7 \\
\hline $1185 \times 1130$ & 5,37 & 128 & 53 & 213 & 113 & 6 & 32 & 11 \\
\hline $1188 \times 1130$ & 5,23 & 125 & 55 & 213 & 114 & 9 & 25 & 28 \\
\hline $1188 \times 1146$ & 5,22 & 125 & 57 & 223 & 123 & 7 & 20 & 16 \\
\hline $1187 \times 1147$ & 5,05 & 121 & 57 & 213 & 125 & 8 & 20 & 3 \\
\hline $\mathrm{H}-5$ & 4,18 & 100 & 57 & 223 & 119 & 4 & 19 & 7 \\
\hline $\mathrm{HB}-83$ & 4,23 & & 57 & 215 & 113 & 6 & 38 & 19 \\
\hline $\mathrm{H}-29$ & 4,82 & & 56 & 223 & 119 & 5 & 19 & 7 \\
\hline
\end{tabular}

Cuadro 4. Medias de rendimiento y características agronómicas de las líneas con mejor ACG a traves de cuatro probadores. PRM, 1991.

\begin{tabular}{|c|c|c|c|c|c|c|c|c|}
\hline \multirow[t]{2}{*}{ LINEA } & \multirow{2}{*}{$\begin{array}{l}\text { Rend. } \\
t / h a\end{array}$} & \multirow{2}{*}{$\begin{array}{l}\text { Días } \\
\text { flor }\end{array}$} & \multirow{2}{*}{$\begin{array}{l}\text { Altura } \\
\text { Plta. }\end{array}$} & \multirow{2}{*}{$\begin{array}{c}(\mathrm{cm}) \\
\mathrm{Maz} .\end{array}$} & \multicolumn{2}{|c|}{ \%azorcas } & \multicolumn{2}{|c|}{$\%$ Acame } \\
\hline & & & & & Desc. & Podr. & Raíz & Tallo \\
\hline 1178 & 6,200 & 56 & 242 & 140 & 2 & 5 & 3 & 1 \\
\hline 1107 & 6,190 & 57 & 247 & 128 & 7 & 6 & 11 & 0 \\
\hline 1130 & 6,030 & 56 & 234 & 126 & 3 & 5 & 2 & 0 \\
\hline 1147 & 6,000 & 55 & 228 & 129 & 7 & 6 & 2 & 1 \\
\hline 1152 & 5,940 & 58 & 240 & 141 & 2 & 6 & 8 & 1 \\
\hline 1146 & 5,940 & 56 & 237 & 132 & 3 & 7 & 10 & 1 \\
\hline 1155 & 5,880 & 56 & 243 & 139 & 7 & 5 & 6 & 0 \\
\hline 1103 & 5,800 & 57 & 235 & 123 & 13 & 9 & 16 & 0 \\
\hline 1111 & 5,760 & 56 & 235 & 122 & 8 & 10 & 7 & 1 \\
\hline \multicolumn{9}{|l|}{ Probadores } \\
\hline \& HB-83M & 6,610 & 58 & 240 & 128 & 1 & 6 & 6 & 1 \\
\hline \& H-29 & 4,140 & 57 & 237 & 126 & 4 & 7 & 16 & 1 \\
\hline \& $\mathrm{H}-5$ & 5,030 & 60 & 258 & 153 & 1 & 6 & 17 & 1 \\
\hline 9 DC-43 & 5,610 & 62 & 247 & 142 & 11 & 14 & 2 & 1 \\
\hline \multicolumn{9}{|l|}{ Testigos } \\
\hline HB-83 & 4,860 & 58 & 238 & 127 & 5 & 7 & 7 & 0 \\
\hline $\mathrm{H}-29$ & 5,580 & 60 & 243 & 137 & 3 & 15 & 21 & 2 \\
\hline $\mathrm{H}-5$ & 4,290 & 60 & 258 & 153 & 1 & 6 & 17 & 1 \\
\hline $\mathrm{HC}-43$ & 4,620 & 59 & 225 & 124 & 10 & 8 & 3 & 1 \\
\hline
\end{tabular}


testigos HB-83, H-29, H-5 y HC-43 en 22, 34, 54 y $35 \%$, respectivamente, y buenas características agronómicas.

Se identificaron 9 líneas con ACG positiva con valores de 0.17-0.61 t/ha, las cuales pueden ser utilizadas para formar nuevas combinaciones híbridas entre ellas mismas o en forma inmediata para formar los híbridos triples identificados.

La líneas que mostró mejor combinación híbrida fue la 1178 derivada del Pool 24.

Cuadro 5. Estimación de aptitud combinatoria general de líneas de maíz grano blanco. PRM, 1991.

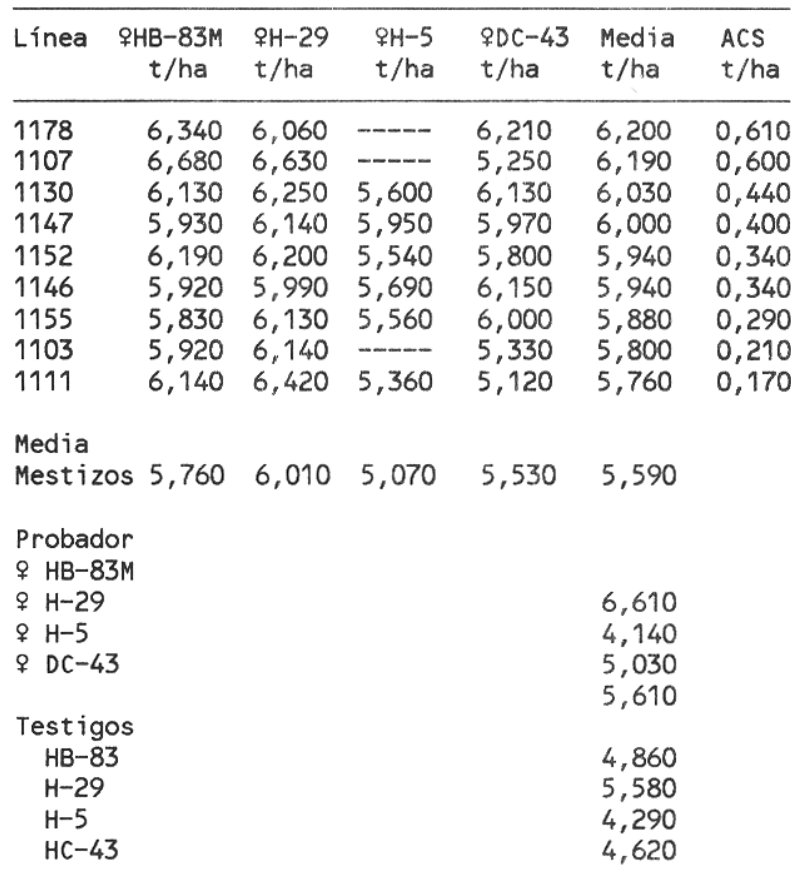

\section{LITERATURA CITADA}

ALLARD, R,W. 1980. Principios de la mejora genética de plantas. Traducción al español por José L. Montoya. Editorial OMEGA, SA. Barcelona. España.

BRAUER, H.O. 1980. Fitogenética aplicada. Editorial LIMUSA, México.

CENTRO INTERNACIONAL DE MEJORAMIENTO DE MAIZ y TRIGO. (CIMMYT), 1989. Programa Regional de Maiz de Cimmyt para Centro America, Panamá y El Caribe. Guatemala.
,1990. Desarrollo y mejoramiento de germoplasma para resistencia a factores adversos bióticos y abióticos. Programa Regional de Maíz. Guatemala. 113 p.

CORDOVA, H. 1990. Estimación de parámetros de estabilidad para determinar la respuesta de híbridos de maíz (Zea mays L.) a ambientes contrastantes de Centro América, Panamá y México. Agronomía Mesoamericana. Vol 2. Año 1991. pag. 1-10.

DOUGLAS, JOHNSON. 1982. Programas de semillas, guias de planeación y manejo. Centro Internacional de Agricultura Tropical (CIAT), Cali, Colombia. Trad. de la 1ra. Edición inglesa.

JUGENHEIMER, R, 1981. Maíz, variedades mejoradas, métodos de cultivo y producción de semilla. México. 112 p.

QUEME, J.L,; SOTO, N.; CORDOV A, H. 1990. Progreso en la Generación de Híbridos de maíz (Zea mays L.), en el Proyecto Colaborativo para Centro América, Panamá y El Caribe. Trabajo presentado en la XIV Reunión de maiceros de la Zona Andina. Septiembre 17-21. Maracay, Venezuela.

\section{RECONOCIMIENTO}

El presente documento es el resultado del trabajo colaborativo de las siguientes entidades e instituciones de Centro América y El Caribe, a quienes se agradece por su valiosa aportación profesional:

Guatemala: Mario Roberto Fuentes, José Luis Queme, Eduardo Landaverry, Carlos Perez, Archelao Avila.(ICTA). Lic. Antonio Cristiani, Hector Deras, Jesús Merino. (Cristiani Burkard).

El Salvador: Adán Aguiluz. (CENTA)

Honduras: Luis Brizuela. (SRN)

Nicaragua: Róger Urbina. (CNIGB)

Costa Rica: Kenneth Jimenez, Carlos Salas, (UCR), Leopoldo Pixley (MAG). 